\title{
AN ABSTRACT FORMULATION OF SOME THEOREMS ON CLUSTER SETS
}

\section{ULYSSES HUNTER ${ }^{1}$}

There are a number of results in the literature which affirm the symmetric structure of functions in terms of Cluster Sets. For example, W. H. Young [5] showed that an arbitrary function $f: R \rightarrow R$ has a remarkable symmetry property which may be stated in terms of Cluster Sets. A real number $y$ is a right limit of $f$ at $x \in R$ if there exists a sequence $x_{n}>x, n=1,2, \cdots$, such that $\lim x_{n}=x$ and $\lim f\left(x_{n}\right)=y$. A left limit of $f$ at $x \in R$ is defined similarly. These sets we designate by $C^{+}(f, x)$ and $C^{-}(f, x)$, respectively. The result of Young asserts that $C^{+}(f, x)=C^{-}(f, x)$ at every $x \in R-A$ where $A$ is a countable set.

Young obtained an analogous result for functions of two variables. By a sector at the origin, we mean either of the closed regions formed by two half-lines emanating from the origin. If $\sigma$ is a sector at the origin, then we designate by $\sigma_{p}$ the image of $\sigma$ under the translation taking the origin into $p$. Let $f: R^{2} \rightarrow R$ be arbitrary and let $p \in R^{2}$. The Cluster Set, $C(f, p)$, of $f$ at $p$ is the set of all real numbers $w$ for which there exists a sequence $\left\{p_{n}\right\} \subset R^{2}$ such that $\lim p_{n}=p$ and $\lim f\left(p_{n}\right)$ $=w$. If $\sigma$ is a sector at the origin, then The Sectorial Cluster Set, $C\left(f, p, \sigma_{p}\right)$, is defined in the obvious manner. The result Young obtained in this case is that

$$
C(f, p)=\bigcap\left[C\left(f, p, \sigma_{p}\right): \sigma_{p}\right]
$$

at every $p \in R^{2}-A$, where $A$ is of the first category and planar measure zero, but not necessarily countable.

Various writers such as H. Blumberg [2], F. Bagemihl [1], P. Erdös and G. Piranian [3], M. Kulbacka [4] and others have studied the properties of Cluster Sets of arbitrary functions and obtained some rather interesting results along these lines. The purpose of this note is to observe a common genesis for certain of the theorems that appear in the papers of Young [5], Erdös and Piranian [3], and Kulbacka [4], after which some examples are given indicating some of the theorems that are involved. We need the following definitions:

Received by the editors September 24, 1964.

1 This represents a portion of the author's Doctoral dissertation in partial fulfillment of the requirements for the Ph.D. at Purdue University. This work was supported in part by NSF Grant 3639-50-395. 
Definition 1. Let $S$ be a set. A collection $M$ of subsets of $S$ is called a $\sigma$-ideal of sets in $S$ if,

(1) $A \in M, B \subset A$ implies $B \in M$,

(2) $A_{n} \in M, n=1,2, \cdots$ implies $\bigcup_{n=1}^{\infty} A_{n} \in M$,

(3) $S \mp M$.

Definition 2. If $S$ is any set and $M$ is a $\sigma$-ideal of sets in $S$, then two topologies $t$ and $t^{\prime}$ are said to be associated modulo $M$ if for every subset $A \subset S$ the $t$ and $t^{\prime}$ derivatives of $A$ differ by a set in $M$.

Definition 3. Let $t$ be a topology on a set $S$ and let $f: S \rightarrow R$ be arbitrary. The $t$-Cluster Set, $C_{t}(f, x)$, of $f$ at $x \in S$ is the set of all real numbers $y$ such that for each $\epsilon>0$,

$$
x \in\left[f^{-1}((y-\epsilon, y+\epsilon))\right]_{t}^{\prime},
$$

(i.e. $x$ is a $t$-limit point of the set $\left.f^{-1}((y-\epsilon, y+\epsilon))\right)$.

Theorem. Let $M$ be a $\sigma$-ideal of subsets of a set $S$ and let $t$ and $t^{\prime}$ be topologies on $S$ which are associated modulo $M$. If $f: S \rightarrow R$ is arbitrary, then $C_{t}(f, x)=C_{t^{\prime}}(f, x)$ at every $x \in S-A$, where $A \in M$.

Proof. We observe that,

$$
\left\{x: C_{t}(f, x) \neq C_{t^{\prime}}(f, x)\right\}=E \cup F
$$

where

$$
F=\left\{x: C_{t^{\prime}}(f, x) \nsubseteq C_{t}(f, x)\right\} \quad \text { and } \quad E=\left\{x: C_{t}(f, x) \nsubseteq C_{t^{\prime}}(f, x)\right\} .
$$

We show $E \in M$. Let $x \in E$. There is a real number $y$ such that $x \in\left[f^{-1}((y-\epsilon, y+\epsilon))\right]_{i}^{\prime}$ for every $\epsilon>0$ and $x \notin\left[f^{-1}\left(\left(y-\epsilon_{0}, y+\epsilon_{0}\right)\right)\right]_{\imath}^{\prime}$, for some $\epsilon_{0}>0$. Thus there are rational numbers, $r_{1}, r_{2}$ with $r_{1}<r_{2}$ such that

$$
x \in\left[f^{-1}\left(\left(r_{1}, r_{2}\right)\right)\right]_{t}^{\prime} \text { and } x \notin\left[f^{-1}\left(\left(r_{1}, r_{2}\right)\right)\right]_{t^{\prime}}^{\prime}
$$

If we let

$$
E_{r_{1}, r_{2}}=\left\{x: x \in\left[f^{-1}\left(\left(r_{1}, r_{2}\right)\right)\right]_{t}^{\prime} \text { and } x \in\left[f^{-1}\left(\left(r_{1}, r_{2}\right)\right)\right]_{t^{\prime}}^{\prime}\right\},
$$

then it follows easily that

$$
E \subset \underset{r_{1}<r_{2}}{\bigcup} E_{r_{1}, r_{2}}
$$

Applying the definition of associated topologies, we see that $E_{r_{1}, r_{2}} \in M$ for every pair of rationals $r_{1}, r_{2}$ and, since $E \subset U_{r_{1}<r_{2}} E_{r_{1}, r_{2}}$, it follows that $E \in M$. A similar argument shows that $F \in M$ which completes the proof of the theorem. 
We would now like to give some examples which exhibit some relationships between the notion of associated topologies and the theory of Cluster Sets.

Example 1. Let $R$ denote the real line. Let $\mu=\{T \subset R: x \in T$ implies there exists an $\eta>x$ such that $[x, \eta) \subset T\}$ and let $\mu^{\prime}=\{T \subset R$ : $x \in T$ implies there exists an $\eta<x$ such that $(\eta, x] \subset T\}$. It can easily be shown that $\mu$ and $\mu^{\prime}$ are topologies on $R$ which are associated modulo the $\sigma$-ideal of countable subsets of $R$. This gives us the theorem of W. H. Young [5]:

TheOREM (W. H. Young). If $f: R \rightarrow R$ is arbitrary and if $C^{+}(f, x)$ and $C^{-}(f, x)$ denote the sets of right and left limits of $f$ at $x$, respectively, then $C^{+}(f, x)=C^{-}(f, x)$ at every $x \in R-A$ where $A$ is a countable set.

ExAmple 2. Let $\tau=\{T \subset R: T$ is measurable and either $T$ is empty or $x \in T$ implies $\left.D_{x}^{+}(T)=1\right\}$ and let $\tau^{\prime}=\{T \subset R: T$ is measurable and either $T$ is empty or $x \in T$ implies $\left.D_{x}^{-}(T)=1\right\}$. (Here the symbols $D_{x}^{-}(T)$ denote the upper right and upper left densities of $T$ at $x$, respectively.) In this case one can show that $\tau$ and $\tau^{\prime}$ are topologies on $R$, which are associated modulo the $\sigma$-ideal of sets of the first category and measure zero. This gives us the following theorem due to M. Kulbacka [4] on the approximate limits of an arbitrary function:

Theorem (M. Kulbacka). Let $f: R \rightarrow R$ be arbitrary. If $w^{+}(x)$ denotes the set of right approximate limits of $f$ at $x$ and $w(x)$ denotes the set of left approximate limits of $f$ at $x$, then the set $\left\{x: w^{+}(x) \neq w^{-}(x)\right\}$ is of the first category and measure zero.

Let $p \in R^{2}$. If $\sigma$ is a sector at the origin, then we designate by $\sigma_{p, \delta}$ the set

$$
\sigma_{p} \cap S(p, \delta)
$$

where $S(p, \delta)$ is the closed disc with center $p$ and radius $\delta>0$.

EXAmple 3. Let $\sigma$ be a sector at the origin and let $\tau$ denote the Euclidean topology on $R^{2}$. If $\tau^{\prime}=\left\{T \subset R^{2}\right.$ : for each $p \in T$ there is an $\epsilon>0$ such that $\left.\sigma_{p, \epsilon} \subset T\right\}$, then the following conditions are satisfied:

(1) $\tau^{\prime}$ is a topology on $R^{2}$.

(2) $\tau$ and $\tau^{\prime}$ are associated modulo the $\sigma$-ideal of sets of the first category and measure zero.

(3) If $\sigma>180^{\circ}$, then $\tau$ and $\tau^{\prime}$ are associated modulo the $\sigma$-ideal of countable sets.

Applying the theorem to this example we get the following theorem of W. H. Young [5]: let $f: R^{2} \rightarrow R$ be arbitrary and let $C(f, p)$ denote 
the Cluster Set of $f$ at $p \in R^{2}$. If $\sigma$ is a sector at the origin, then we designate by $C\left(f, p, \sigma_{p}\right)$ the Sectorial Cluster Set of $f$ at $p$.

Theorem (W. H. Young). For every $f: R^{2} \rightarrow R$,

$$
C(f, p)=\cap\left[C\left(f, p, \sigma_{p}\right): \sigma_{p}\right]
$$

at every $p \in R^{2}-A$, where $A$ is of the first category and planar measure zero, but not necessarily countable.

Also, in connection with Example 3, we would like to mention that in [2], H. Blumberg pointed out that if the angles of the sectors considered exceed $180^{\circ}$, then the countable character of the exceptional set $A$ is restored.

\section{REFERENCES}

1. F. Bagemihl, Curvilinear cluster sets of arbitrary functions, Proc. Nat. Acad. Sci. U.S.A. 41 (1955), 379-381.

2. H. Blumberg, Exceptional sets, Fund. Math. 32 (1939), 1-32.

3. P. Erdös and G. Piranian, Restricted cluster sets, Math. Nachr. 22 (1960), 155158.

4. M. Kulbacka, Sur l'ensemble des points de l'asymetrie approximative, Acta Sci. Math. (Szeged) 21 (1960), 90-95.

5. W. H. Young, La symetrie de structure des fonctions de variables réelles, Bull. Sci. Math. (2) 52 (1928), 265-280.

California State College at Hayward 